\title{
Division of methods for counting helminths' eggs and the problem of efficiency of these methods
}

\author{
Katarzyna Jaromin-Gleń1, Teresa Kłapeć ${ }^{2}$ Grzegorz Łagód ${ }^{3}$, Jacek Karamon ${ }^{4}$, Jacek Malicki', \\ Agata Skowrońska ${ }^{3}$, Andrzej Bieganowski ${ }^{1}$ \\ ${ }^{1}$ Institute of Agrophysics, Polish Academy of Sciences, Lublin, Poland \\ ${ }^{2}$ Department of Biological Health Hazards and Parasitology, Institute of Rural Health, Lublin, Poland \\ ${ }^{3}$ Department of Water Supply and Wastewater Disposal, Faculty of Environmental Engineering, Lublin University of \\ Technology, Lublin, Poland \\ ${ }^{4}$ Department of Parasitology and Invasive Diseases, National Veterinary Research Institute, Pulawy, Poland
}

Jaromin-Gleń K, Kłapeć T, Łagód G, Karamon J, Malicki J, Skowrońska A, Bieganowski A. Division of methods for counting helminths' eggs and the problem of efficiency of these methods. Ann Agric Environ Med. 2017; 24(1): 1-7. doi: 10.5604/12321966.1233891

\section{Abstract}

From the sanitary and epidemiological aspects, information concerning the developmental forms of intestinal parasites, especially the eggs of helminths present in our environment in: water, soil, sandpits, sewage sludge, crops watered with wastewater are very important. The methods described in the relevant literature may be classified in various ways, primarily according to the methodology of the preparation of samples from environmental matrices prepared for analysis, and the sole methods of counting and chambers/instruments used for this purpose. In addition, there is a possibility to perform the classification of the research methods analyzed from the aspect of the method and time of identification of the individuals counted, or the necessity for staining them. Standard methods for identification of helminths' eggs from environmental matrices are usually characterized by low efficiency, i.e. from $30 \%$ to approximately $80 \%$. The efficiency of the method applied may be measured in a dual way, either by using the method of internal standard or the 'Split/Spike' method. While measuring simultaneously in an examined object the efficiency of the method and the number of eggs, the 'actual' number of eggs may be calculated by multiplying the obtained value of the discovered eggs of helminths by inverse efficiency.

\section{Key words}

helminths' eggs, parasitological methods, sewage sludge, biosolid matrices, efficiency of method

\section{INTRODUCTION}

Helminths belong to the most common infectious agents which still affect human health in both rural and urban areas. The problem of helminths infection afflicts especially the developing countries of the tropical zone where, unfortunately, insufficient attention is paid to sanitaryhygienic conditions [1-3]. Helminths may exert a hazardous effect on the host as a mechanical, chemical and biotic agent; therefore, they may damage the intestinal wall, erythrocytes, muscle tissue and internal organs (lungs, liver, kidneys, and brain). Substances secreted by the parasite may also have a toxic or allergic effect on the host $[4,5]$. The complications developing as a result of infection favour the development of other diseases, such as malaria, tuberculosis or AIDS, and contribute to the decrease in the effectiveness of vaccines $[2,6-8]$.

Sanitary control of wastewater and sewage sludge concerns mainly the developed countries. When the results of control are positive, examined objectives may be applied for agricultural or natural use $[9,10]$, otherwise they can be utilized in other branches of the economy $[11,12]$. Unfortunately, in the developing countries - especially those struggling with a deficit of fresh water - wastewater and

Address for correspondence: GrzegorzŁagód, Faculty of Environmental Engineering, Lublin University of Technology, Nadbystrzycka 40B Str., 20-618 Lublin; Poland E-Mail: g.lagod@pollub.pl (G.Ł)

Received: 30 November 2015; accepted: 1 March 2016; first published on January 2017 sludge are frequently used without any supervision, even for watering or fertilization of fruit and vegetable crops which are consumed raw [13-16].

Considering the importance of examinations for the presence of helminths' eggs, it is not surprising that a great effort by many research teams is biased towards the constant improvement of measurement techniques for the detection and counting of the eggs of these parasites. A review of the literature may lead to a certain sense of chaos, and cause difficulties in the selection of the most optimal method for the investigations/measurements. Unfortunately, in literature reports, there may still be found authors who report the results obtained while counting eggs; however, without any information concerning the efficiency of the method used in their laboratories. Such result cannot in any way be interpreted in general categories, nor is it possible to compare them with results obtained in other laboratories. In order to make the reader familiar with the problem of validation, the final section of the report is devoted to this problem.

\section{OBJECTIVE}

The primary aim of the presented study is the description of the possible classification of methods for counting helminths' eggs (according to various criteria). The second goal of the study is a discussion of the problem of intra-laboratory validation of the methods of counting helminths' eggs. 
Basic division of diagnostic techniques. Techniques applied in the detection of the eggs of intestinal parasites in various types of matrices may generally be divided into qualitative, which only serve to determine if the parasites which are sought are present in the sample examined, and quantitative methods, which enable the estimation of the number of these parasites. The quantitative methods are used in medical and veterinary diagnostics, as well as in sanitary engineering (e.g. while investigating sewage sludge or wastewater from health care facilities) for the assessment of intensity of parasite invasion, or the possibilities to use waste water and sewage sludge; therefore, in a further section of this report the authors focus on these methods.

Irrespective of the type of method applied in the analysis (qualitative or quantitative), it may be generally divided into two stages - appropriate preparation of samples for analysis, and sole analysis using microscopic methods. Usually, in order to obtain the most precise result of examination, the methods for the preparation of samples are expanded by the subsequent stages/mechanical procedures, and the additions of proper solutions are applied - most often floating. Also, the counting of the developmental forms is carried out in special chambers, the construction of which is frequently complicated.

In the description of older methods of counting developmental forms of parasites, mainly the eggs of helminths, the emphasis was placed on the way of preparation of the sample for analysis, whereas the process of counting under the light microscope was less precisely described. At present, newer methods approach the entire process of seeking results as a whole, from the moment of samples preparation, through counting in proper chambers, to the conversion into the desired units by means of appropriate correction coefficients.

Figure 1 presents the general scheme of the division of the selected quantitative methods of counting the eggs of helminths and other developmental forms of parasites (oocysts, cysts, entire parasites). Here, the method of sample preparation was adopted as the basic criterion of division. Such an approach seems to be justified, because this stage, to a great extent, decides about the size of error which is always committed while counting eggs. The techniques of counting are also considered here; however, in the presented division they play a secondary role.

Division of methods for counting helminths' eggs according to the way of sample preparation. As shown in Figure 1, quantitative methods may be divided into:

- measurements in non-densified samples (usually subjected to homogenization only;

- measurements in densified samples (concentrated).

Methods in which the techniques densifying the developmental forms of parasites are not applied are, among

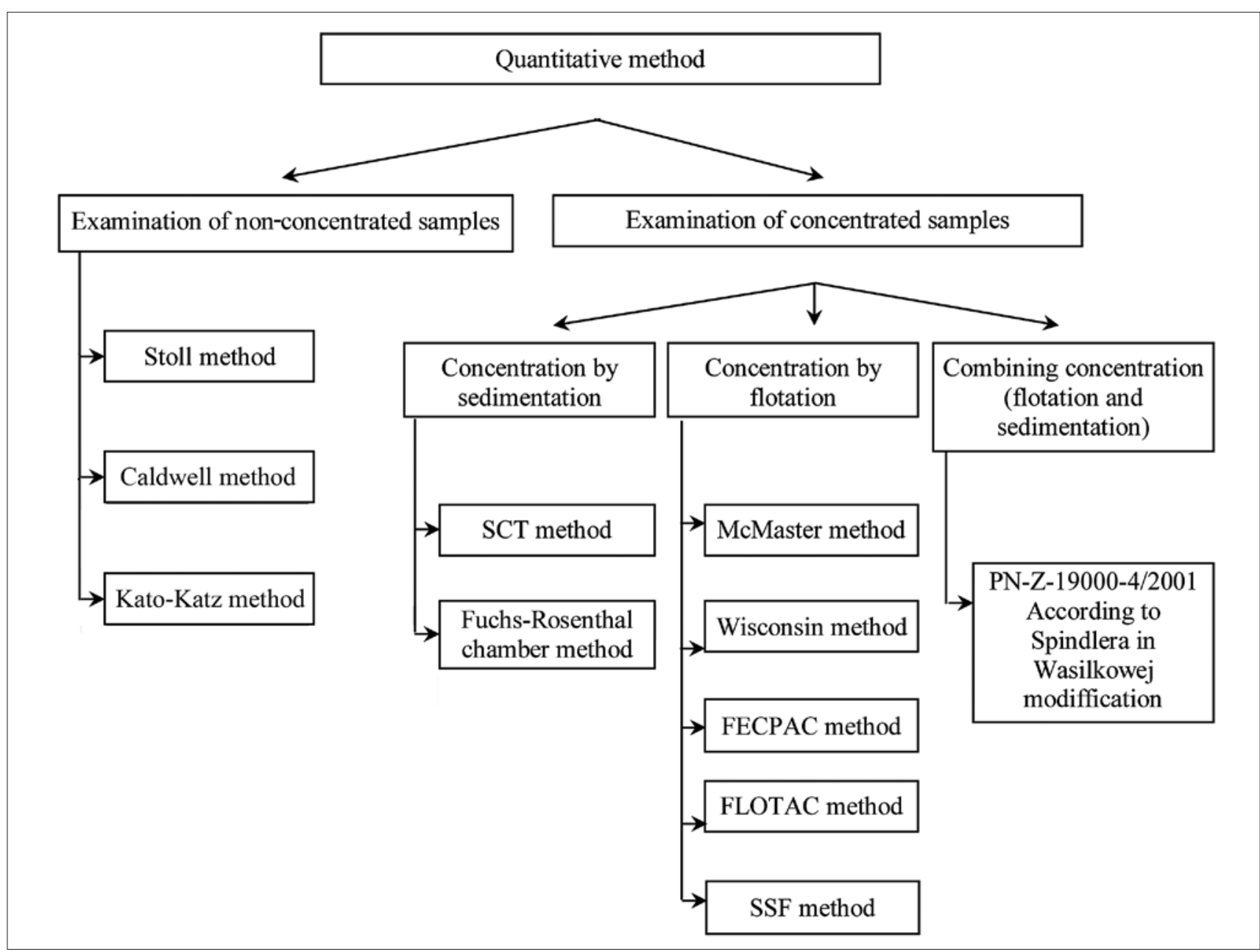

Figure 1. Scheme of division of quantitative methods for calculation of helminths' eggs (and other developmental forms of parasites) according to the way of preparing samples (with exemplary methods from individual groups) 
others, the Stoll method, Caldwell method $[17,18]$ and KatoKatz method [19-21].

Methods with a concentration of parasite forms may be divided into those based on sedimentation techniques, flotation techniques, and methods which are the combination of both these techniques in a proper order.

The principle of functioning of sedimentation methods consists in the application of liquids of a density lower than the density of the sought forms of parasites. The eggs of parasites, as well as the cysts of protozoa, are separated from contaminants and, being heavier, fall to the bottom of the vessel, and the densification increases the probability of detection of parasite forms.

The principle of the functioning of flotation methods consists in the application of liquids of a density higher than that of the parasite eggs. Due to this, the eggs 'become lighter' and rise to the surface of the solution.

Considering the fact that at present there is no method for the determination of eggs of helminths in sewage sludge, the standardized flotation method by Spindler is used, as modified by Wasilkowa, designed mainly for the examination of soil, and the flotation method by Quinn et al. (1980). The number of eggs is converted into $1 \mathrm{~kg}$ dry mass of the sample $[22,23]$.

Criteria of selection of sample preparation method. Selection of the method of preparation of the sample used for counting the developmental forms of parasites (most frequently eggs) depends on many factors. The most important of these factors are primarily the species of the parasites sought - some parasite eggs, e.g. Toxocara spp., possess surfaces which cause their adhesion to devices and instruments applied for the preparation of samples for analysis $[24,25]$. Also, some techniques of sample preparation may cause damage to the analysed and counted forms. For example, the use of flotation or disperging liquids may cause damage or deformations which considerably hinder the counting, while leaving the sample for a longer time at the room temperature (for sedimentation) may result in the hatching of the larvae designed for counting (e.g. hatching of larvae of strongyles). In addition, some forms (eggs) have a sufficiently high specific gravity (e.g. the eggs of trematodes), that their efficient density is possible only by using sedimentation methods [18].

Selection of the method, to a large extent, depends also on the matrix examined, where the forms of parasites are counted. Thus, according to the consistency of samples for examination (solid, paste-like, semi-liquid or liquid), and the type and size of particles in a solid phase present in these samples (e.g. considerable amounts of fat, fibrous elements, coagulants used for the dewatering of sewage sludge), various flotation solutions and supporting substances may be applied.

A good example of the consequences of inappropriate (in this case, to the matrix in which measurement is performed) preparation of the sample for analysis may be the fact that until the end of the 1990s in Poland, the eggs of intestinal parasites were rarely detected in sewage sludge. This does not mean the absence of these eggs. The cause of low detectability of the eggs is the fact of introduction into the process of dewatering of sewage sludge (a stage in the process of sewage processing) of polyelectrolytes (playing the role of flocculants). These are chemical substances which cause a reduction in the amount of sewage sludge supporting their densification and clumping. This results in 'imprisonment' of the eggs of intestinal parasite in sludge.

However, recently, the new, efficient method for examination of sewage sludge containing flocculants was elaborated. Thanks to using the successive stages of mixing, sedimentation and flotation this method detects nematode eggs more effectively [26].

Division of methods according to the way of counting helminths' eggs under a microscope. The element of the procedure, which should be considered while analyzing and classifying methods for detection/counting developmental forms of parasites, are the final methods of identification and counting. These methods are based on microscope observations of appropriately prepared specimens - the problem mentioned above. The counting usually takes place under a light microscope in transmitted light, most often in a bright field of vision.

Division according to the device for sample analysis under a microscope. The stage of counting developmental forms of parasites may be classified with respect to the application of various devices for sample analysis under the microscope. In this case, the following instruments may be distinguished:

- classic microscope glass slides and cover slips;

- general use chambers - e.g. Burker, Thoma, FuchsRosental, etc.;

- parasitology chambers - e.g. McMaster, FLOTAC, FECPAC.

The above-presented division shows that microscopic observations may be performed on ordinary microscope glass slides - e.g. in the Stoll's [17], Caldwell's [18] or Wisconsin methods [21,27]; also, this stage may be performed in appropriate chambers. For this purpose, chambers of general use are applied, e.g. Fuchs-Rosenthal (which is also used in cytology, haematology, microbiological and protozoological examinations) [28, 29], Sedgwick-Rafter [30,31], or Doncaster chambers - most often used in hydrobiology and sanitary engineering, more rarely in veterinary medicine while counting developmental forms of parasites - mainly the eggs of helminths [32].

However, the counting of developmental forms of parasites is most frequently performed in chambers specially elaborated for parasitological examinations. An example of such chambers may be the most commonly known: McMaster [18,33], FLOTAC [18, 34], and FECPAC [18, 35]. These chambers occur in various modifications, which usually consist in an increase (more rarely decrease) of the working volume, obtained most often by the addition of extra calculation sub-chambers. In their basic variants the described chambers usually consist of two or three subchambers. Therefore, it would be more correct to state that the described chambers occur in various volume variants and number of sub-chambers. This, in fact, results in an increase in the volume of the sample analyzed - which, on the one hand is related with the prolongation of the working time, while on the other hand, provides a higher precision of the outcome. Parasitological chambers may also use the phenomena facilitating identification and counting, e.g. flotation support in the FECPAC chamber.

The task of a chamber is such an exposure of the sample which enables the counting of the eggs (assuming all are 
present in the sample) in a precisely determined volume of the sample. This is possible by the provision of a sufficiently thin layer of the sample to enable the observation of all the eggs. It is important to count the eggs on the largest surface possible. In order to facilitate this task, special lines or meshes are applied in chambers, which are the element of reference in space, minimizing, at the same time, the risk of omitting the counted object or its multiple consideration. Usually, such chambers are divided into two or three segments of equal volume, which may be approached as calculation subchambers.

However, the use of chambers for counting parasites' forms also has disadvantages, the most important of which is that with an increased thickness of the chamber in relation to the microscope glass slides and cover slips, not all lenses may be applied. This is due to an insufficient working distance at magnification greater than $\mathrm{x} 20$, which often hinders identification of the objects examined [18].

Division according to method of identification of parasite eggs. The subsequent division may be performed based on methods for identification of developmental forms of parasites. Here, two basic methods may be mentioned:

- Direct observations under a light microscope. The advantage of such an analysis is the possibility of changing magnification, contrast or/and method of observation and, in the last resort, the physical moving of the analysed sample in order to make sure that what is observed under the microscope is what is sought for. The disadvantage is the necessity to observe time regimes, i.e. the analysis cannot be prolonged or postponed in time.

- The performance of digital photographs and their later analysis. In this case, the disadvantage is the lack of possibility to change the exposure of the sample, and in the case of doubts, it is not possible to return to the sample. Simultaneously, such an approach provides: 1) the possibility to perform analysis at any time, and 2) considerably facilitates consultations with more experienced diagnosticians. In addition, it is possible to apply support in the form of software for image analysis which, although not yet sufficiently specialized to count eggs perfectly, allows preliminary selection of images. Attempts to use such a solution were undertaken during, e.g. identification of species affiliation of oocysts of the coccidia of the genus Eimeria occurring in pigs [36]. Nevertheless, today, the use of such an approach in a wider parasitological diagnostics seems to be rather a further perspective.

Obviously, there is a possibility to combine the two abovementioned methods. Such an approach has an additional advantage in that it allows education of both the staff performing measurements, and so-called learning of the software. This means the possibility to indicate in the image of the object - here, the eggs of helminths - identified in the visual measurement.

Staining of specimens. Identification of the majority of helminths' eggs is based on the detection of the eggs in the sample examined. Most frequently, helminths' eggs are identified based on their characteristics in fresh faeces, these characteristics are: size, shape, thickness and colour of the eggshell, presence of the developmental stage and other traits, such as lid-like operculum or plugs, spines, knode-like bumps or thickenings, outer and inner coats, and fibrils. During identification, the eggs of worms do not require staining, and sometimes as a result of staining they become damaged or too intensively stained, and in such a situation are generally difficult to identify. However, on the other hand, staining may facilitate the observation of the eggs in the sample examined.

Parasitological specimen may be stained with Lugol's solution. The advantage of this method is the staining of the inner structure of worm eggs, while the disadvantage is that an excessive concentration of the Lugol's iodine solution (higher than $1 \%$ solution) causes the death of larvae of the worms, the eggs are visible, but their structure is obscured [19].

Another technique, which allows the obtaining of direct, brightened and stained smear from fresh faeces is the method by Kato-Katz. This method serves the detection of helminths' eggs and larvae of worms (cellophane as cover strips soaked in glycerol-malachite green or methylene blue solution). The advantage of this method is the examination of a larger amount of faeces in one specimen, which increases the probability of the detection of parasites [19, 20,37].

The results of experiments carried out by Dąbrowska et al. (2014) confirmed a high usefulness of the LIVE/DEAD commercial assay stain kit for staining the eggs of intestinal parasites, live or dead, in sewage sludge [38]. The abovementioned stain kit is used basically in microbiological studies for the assessment of the viability of bacterial cells. With respect to sewage sludge, it was found that live eggs of Ascaris spp., Trichuris spp. and Toxocara spp. stained with the LIVE/DEAD assay, under the fluorescent microscope emit green light, while dead eggs - red light. Trials with the use of this kit in the estimation of viability of eggs have also been described by other authors [39]. The main advantage of this test is that it is an alternative with respect to time consuming and subjective observation of the eggs during incubation, whereas its disadvantage is the high cost.

Correction coefficients of counting results. The number of parasite eggs counted in the examined sample does not in any way provide the ultimate result. Due to the lack of reference to unit of mass and lack of consideration of efficiency of the method, the results are not comparable to those obtained in other laboratories.

Thus, the first stage of processing of the results is the reference to the unit of mass. Theoretically, it is possible to refer to the unit of volume; however, considering different densities of various matrices, the conversion into the unit of volume could result in considerable errors in interpretation.

Correlation coefficients allow conversion of the obtained result of microscopic observations into the basic unit, which is usually $1 \mathrm{~g}$ of raw sample, or dry mass. This coefficient is generally adopted according to the procedure of sample preparation - amount of the raw sample examined and added solutions (for homogenization, flotation, etc.), and the volume of the sole chamber, as well as the number of segments/ sub-chambers analyzed during measurements. However, if, while calculating the coefficient, the humidity of the matrix is not considered (this concerns matrices of solid or dense suspension consistency) or concentration (with respect to liquid matrices), the reference of the result to raw sample mass does not provide an unequivocal result, and may be the source of serious errors in interpretation. 
Efficiency of method. An important problem mentioned previously which, unfortunately, is not reflected in reports, is the efficiency of the method. The necessity for considering efficiency while providing the ultimate outcome of analysis of helminths' eggs results from the fact that the effectiveness of counting is not a hundred percent, and depends primarily on: species/genus of a parasite, matrix, method of sample preparation (including concentration), technique of counting, infrastructure used, and experience of the person performing the counting. The above-mentioned specifications do not exhaust the complete list of the sources of uncertainty which, in individual cases, should be considered at the stage of validation of the method in each laboratory.

Some researchers provide various definitions and terms of efficiency; therefore, the following definition may be adopted to provide explicitness:

$$
\text { efficiency }=\frac{\text { counted number of eggs }}{\text { actual number of eggs }} \cdot 100 \%
$$

While the counted number of eggs is the available information, because it is the result of direct counting of eggs in a sample, the obtaining of information concerning the actual number of eggs requires an additional measurement. As previously stated, it is practically impossible to determine this number in a direct way. Although the situation may be imagined when the matrix is diluted, all aggregates ground or crushed, and due to these procedures all helminths' eggs are released and become visible and possible to identify by the person performing the counting. However, anyone who has physically performed such measurements would admit that this is purely a theoretical possibility. If so, another way of estimation of the value of actual number of eggs should be sought for.

Analysis of data from literature leads to the conclusion that the methods and their modifications presented in many reports may be basically reduced to one approach, based on the assumption that a reliable evaluation of the efficiency of the method is possible, when validation counts are performed on the material with a known number of parasitic forms. In other words, the efficiency of the method may be assessed by the enrichment of the sample with a known number of helminths' eggs. However, the performance of this assumption may differ considerably.

Before discussing in detail the methods of estimation of the efficiency of counting, it is worth presenting another approach to this problm. According to some authors [40,41], if there is an absence of a true gold standard, meta-analysis can be applied, based on the comparison of the sensitivity and the quantitative performances of several methods. In their study, Nikolay et al. (2014) [41] mentioned the following methods: Kato-Katz, direct microscopy, McMaster, FLOTAC and Mini-FLOTAC. The researchers concluded that the highest sensitivity was for the FLOTAC method and the lowest for the direct microscopy method. Such a result could have been expected, considering the method of the preparation of samples and the method of measurement. However, a disadvantage of the approach based on meta-analysis is the statistical evaluation of methods - not a particular analysis, performed in a particular laboratory. Evaluation of individual analysis is possible only when the internal standard method is used.

Indirect method. The simplest procedure which, however, is not always possible to perform, is the enrichment (contamination) of a clean matrix with helminths' eggs which are to be counted on this matrix. Knowing how many eggs were implemented and how many were counted, it is easy to calculate the efficiency of the method - directly from Formula 1. The efficiency calculated in this way may be referred to the actual measurements $[25,29]$. The carrying out of validation studies by this method allows verification of the theoretical assumptions applied in popular quantitative methods, such as, e.g. McMaster's method which has been used for many decades [18], and the FLOTAC method introduced relatively recently [42], as well as assessment of the actual limitations of the method considered as a 'gold standard' in the diagnostics of echinococcosis in animals [43]. The problem is the necessity for obtaining exactly the same matrix on which eggs will be counted in the actual measurement, but totally free from parasites. For the needs of the presented report, the method has been named 'indirect', because the efficiency is determined in a sample other than that examined, and then referred to the examined sample.

'Split/Spike' method'. The second method (also occurring in various modifications) is the addition of a known number of eggs to a naturally contaminated matrix [3]. This method requires counting the eggs twice. At the first stage, the eggs are counted in the actual samples. Subsequently, to similar samples (not the same because the samples after the first stage are suitable only for utilization), a known number of eggs is added, and the counting procedure is repeated. This method required a theoretical assumption of homogeneity of the matrix in which the eggs are counted, i.e. it is assumed that in a specified mass (volume) the same numbers of eggs are present, which is difficult to obtain in the case of parasites. Thus, an increase in the number of eggs in the enriched sample provides information about how many of the added eggs have been counted. The way of counting with the discussed procedure is as follows: (note: the formulae presented below do not take into consideration the conversion of the number of eggs into dry mass/sample volume - the conversion refers to the mass/volume collected for examination):

$$
\begin{gathered}
\text { efficiency }=\frac{\mathrm{x}_{2}-\mathrm{x}_{1}}{\mathrm{y}} \cdot 100 \% \\
\mathrm{x}_{0}=\frac{\mathrm{y}}{\mathrm{x}_{2}-\mathrm{x}_{1}} \cdot \mathrm{x}_{1}
\end{gathered}
$$

where:

$\mathrm{x}_{0}$ - actual number of eggs in the sample examined;

$\mathrm{x}_{1}$ - number of eggs counted in the first sample examined;

$\mathrm{x}_{2}$ - number of eggs counted in the second sample enriched after addition of a known number of eggs;

$y$ - number of eggs added to the sample.

Method of addition of marked inner pattern. The final method discussed consists in addition to the sample of a marked pattern with which helminths' eggs are stained [29]. The advantage of this method is the fact that the counting of eggs in the examined sample is performed once (obviously, the stained and not stained eggs are counted). An additional benefit of this method is that in the case of its use there is no need to densify the sample (i.e. to apply previously-mentioned methods of flotation, sedimentation, etc.); however, the best possible homogenization is recommended. 
The way of calculating using this method is presented by the following formula:

$$
\begin{gathered}
\text { efficiency }=\frac{\mathrm{y}}{\mathrm{Y}} \cdot 100 \% \\
\mathrm{x}_{0}=\frac{\mathrm{Y}}{\mathrm{y}} \cdot \mathrm{x}
\end{gathered}
$$

where:

$\mathrm{x}_{0}$ - actual number of eggs in the sample examined;

$\mathrm{x}$ - number of colourless eggs counted in the sample examined;

$y$ - number of stained eggs counted in the sample examined; $\mathrm{Y}$ - number of stained eggs added to the sample.

\section{SUMMARY}

The presented divisions are an attempt to put in order the wide spectrum of measurement procedures related with the identification and counting of helminths' eggs. Obviously, the selection of an individual method (or its modification) will depend, among other things, on the examined matrix, experience, preferences of the analyst and the infrastructure possessed. However, before making a choice, it is worthwhile becoming familiar with the entire spectrum of possibilities.

Generally speaking, the methodology should be selected in such a way that the preparation of the sample does not cause loss in quantity, or, e.g. damage or hatching of the eggs. The methods of counting and the type of chamber, if they are designed for parasitology, are less important - usually the larger the volume, the higher the efficiency of the method which; however, is associated with a greater outlay of time and work. In addition, with respect to the chambers, it may be presumed that if the developmental forms have a lower density, the chambers with flotation work well.

Irrespective of the method, it should be unequivocally stated that from the aspect of the quality of studies (reliability of the result) the preparation of the sample and determination of the efficiency of counting are the most important. Here, according to the methods of sample preparation used, possibilities to obtain eggs as an inner pattern, and the preferences of the person performing the examinations, several methods for the estimation of efficiency may be indicated. Literature review shows that the efficiency of methods of identification of helminths' eggs depends on the procedures and their modifications applied, and not the fact that the higher the efficiency the better the method or laboratory assistant. Irrespective of the value of efficiency, when reliably estimated, it enables the determination of the actual number of eggs in the examined sample. This result, together with the provided efficiency of the method, should be presented in publications and study reports.

\section{CONCLUSIONS}

- For sanitary and epidemiological reasons it is important to recognize the number of helminths' eggs in various objects - environmental matrices.

- Irrespective of the procedure applied, examined object, etc., the methods applied in the determination of the number of eggs are always burdened with considerable errors resulting from the difficulties with identification from the sample, and obtaining all eggs in a pure suspension.

- These errors may be estimated by determination of the efficiency of the method used, among other things, by using the inner pattern, or 'Split/Spike' methods.

- If, during the examinations, the number of eggs and efficiency of the method are measured simultaneously, the 'actual' number of eggs may also be calculated.

- While presenting the results of studies it is recommended to provide information concerning the volume density and humidity, which would allow the conversion of the number of eggs into volume of unit of mass (or the opposite).

\section{Acknowledgements}

This paper was partly financed from the funds of the National Science Centre, Poland (No.2015/17/N/ST10/02194) and Ministry of Science and Higher Education in Poland within the statutory research number S-70/WIŚ/2016

\section{REFERENCES}

1. de Silva NR, Brooker S, Hotez PJ, Montresor A, Engels D, Savioli L. Soil-transmitted helminth infections: updating the global picture. Trends Parasitol. 2003; 19(12): 547-551.

2. Bethony J, Brooker S, Albonico M, Geiger SM, Loukas A, Diemert D, Hotez PJ. Soil-transmitted helminth infections: ascariasis, trichuriasis, and hookworm. Lancet. 2006; 367(9521): 1521-1532.

3. Pullan RL, Smith JL, Jasrasaria R, Brooker SJ. Global numbers of infection and disease burden of soil transmitted helminth infections in 2010. Parasites Vector. 2014; 7(37): 1-19. doi: 10.1186/1756-3305-7-37.

4. Palmer LJ, Celedon JC, Weiss ST, Wang B, Fang Z, Xu X. Ascaris lumbricoides infection is associated with increased risk of childhood asthma and atopy in rural China. Am J Resp Crit Care. 2002; 165(11): 1489-1493.

5. Ojha SC, Jaide C, Jinawath N, Rotjanapan P, Baral P. Geohelminths': public health significance. J Infect Dev Countr. 2014; 8(1): 5-16.

6. van Riet E, Hartgers FC, Yazdanbakhsh M. Chronic helminth infections induce immunomodulation: consequences and mechanisms. Immunobiology. 2007; 212(6): 475-490.

7. World Health Organization. Research Priorities for Helminth Infections. Technical Report of the TDR Disease Reference Group on Helminth Infections.Geneva, 2013.

8. Zeukeng F, Tchinda VHM, Bigoga JD, Seumen CHT, Ndzi ES, Abonweh G, Makoge V, Motsebo A, Moyou RS. Co-infections of malaria and geohelminthiasis in two rural communities of Nkassomo and Vian in the Mfou Health District, Cameroon. PLoS Neglected Tropical Diseases. 2014; 8(10): e3236. doi:10.1371/journal.pntd.0003236.

9. Mantovi P, Baldoni G, Toderi G. Reuse of liquid, dewatered, and composted sewage sludge on agricultural land: effects of long-term application on soil and crop. Water Res. 2005; 39(2-3): 289-296. doi: 10.1016/j.watres. 2004.10.003

10. Frąc M, Oszust K, Lipiec J, Jezierska-Tys S, Oluchi Nwaichi E. Soil microbial functional and fungal diversity as influenced by municipal sewage sludge accumulation. Int J Environ Res Public Health. 2014; 11 : 8891-8908; doi: 10.3390/ijerph110908891

11. Werle S. Sewage sludge-to-energy management in eastern Europe: a Polish perspective. Ecol Chem Eng S. 2015;22(3): 459-469. doi: 10.1515/ eces-2015-0027

12. Franus M, Barnat-Hunek D, Wdowin M. Utilization of sewage sludge in the manufacture of lightweight aggregate. Environ Monit Assess. 2016; 188 (1): e10. doi: 10.1007/s10661-015-5010-8

13. Kozan E, Gonenc B, Sarimehmetoglu O, Aycicek H. Prevalence of helminth eggs on raw vegetables used for salads. Food Control. 2005; 16(3): 239-242.

14. Mahvi AH, Kia EB. Helminth eggs in raw and treated wastewater in the Islamic Republic of Iran. East. Mediterr. Health J. 2006; 12(1-2): $137-143$.

15. Koné D, Cofie O, Zurbrügg C, Gallizzi K, Moser D, Drescher S, Strauss $\mathrm{M}$. Helminth eggs inactivation efficiency by fecal sludge dewatering and co-composting in tropical climates. Water Res. 2007; 41(19): 4397-4402. 
16. Abougrain AK, Nahaisi MH, Madi NS, Saied MM, Ghenghesh KS. Parasitological contamination in salad vegetables in Tripoli-Libya. Food Control. 2010; 21(5): 760-762.

17. Stoll NR. Investigations on the control of hookworm disease. XV. An effective method of counting hookworm eggs in feces. Amer J Hygiene. 1923; 3: 59-70.

18. Kochanowski M, Karamon J, Dąbrowska J, Cencek T. Coproscopical quantitative methods in the parasitological diagnosis - the use and problems with estimation of their efficiency (in Polish). Postep Mikrobiol. 2013; 52(1): 111-118.

19. World Health Organization. Basic Laboratory Methods in Medical Parasitology. Geneva, 1991.

20. Santos FLN, Cerqueira EJL, Soares NM. Comparison of the thick smear and Kato-Katz techniques for diagnosis of intestinal helminth infections. Rev Soc Bras Med Tro. 2005; 38(2): 196-198.

21. Goodman D, Haji HJ, Bickle QD, Stoltzfus RJ, Tielsch JM, Ramsan M, Savioli L, Albonico M. A comparison of methods for detecting the eggs of Ascaris, Trichuris, and hookworm in infant stool, and the epidemiology of infection in Zanzibari infants.Am J Trop Med Hyg. 2007; 76(4): 725-731.

22. Quinn R, Smith HV, Bruce RG, Gidwood RWA. Studies on the incidence of Toxocra and Toxocara spp. ova in the environment. I. A comparision of flotation procedures for recovering Toxocara spp. ova from soil. J Hyg. 1980; 84(1): 83-89.

23. PN-Z-19000-4 2001. Polish Standard. Soil quality - Assessment of the soil sanitary conditions - Detection of eggs of the intestinal parasites Ascaris lumbricoides and Trichuris trichiura.

24. Overgaauw PAM, van Knapen F. Toxocarosis, an important zoonosis. EJCAP. 2008; 18(3): 259-266.

25. Kochanowski M, Dąbrowska J, Karamon J, Cencek T, Osiński Z. Analysis of the accuracy and precision of the McMaster method in detection of the eggs of Toxocara and Trichuris species (Nematoda) in dog faeces. Folia Parasitol. 2013; 60(3): 264-272.

26. Zdybel J, Cencek T, Karamon J, Kłapeć T. Effectiveness of selected stages of wastewater treatment in elimination of eggs of intestinal parasites. Bull Vet Inst Pulawy. 2015; 59: 51-57.

27. Cox DD, Todd AC. Survey of gastrointestinal parasitism in Wisconsin dairy cattle. J Am Vet Med Assoc. 1962; 141: 706-709.

28. Varga I, Sreter T, Békési L. Quantitative method to assess Cryptosporydium oocyst shedding in the chicken model. Parasitol Res. 1995; 81(3): 262-264.

29. Malicki J, Montusiewicz A, Bieganowski A. Improvement of counting helminth eggs with internal standard. Water Res. 2001; 35(9): 2333 2335.

30. Gallizzi K. Co-composting reduces helminth eggs in fecal sludge, a field study in Kumasi, Ghana, SANDEC Switzerland http://www.eawag.ch/
fileadmin/Domain1/Abteilungen/sandec/publikationen/SWM/Cocomposting/Gallizzi_2003.pdf (access:2015.11.03).

31. Cobbina SJ, Kotochi MC, Korese JK, Akrong MO. Microbiological contamination in vegetables at the farm Gate Due to irrigation with wastewater in the Tamale Metropolis of Northern Ghana. JEP. 2013; 4(7): 676-682.

32. Marley CL, Cook R, Barrett J, Keatinge R, Lampkin NH, McBride SD. The effect of dietary forage on the development and survival of helminth parasites in ovine faeces, Vet Parasitol. 2003; 118(1-2): 93-107.

33. Pereckiene A, Petkevicius S, Vysniauskas A. Comparative evaluation of efficiency of traditional McMaster chamber and newly designed chamber for the enumeration of nematode eggs. Acta Vet Scand. 2010; 52(Suppl 1): S20.

34. Cringoli G, Rinaldi L, Maurelli MP, Utzinger J. FLOTAC: new multivalent techniques for quantitative copromicroscopic diagnosis of parasites in animals and humans. Nat Protoc. 2010; 5(3): 503-515.

35. Presland SL, Morgan ER, Coles GC. Counting nematode eggs in equine faecal samples. Vet Rec. 2005; 156(7): 208-210.

36. Plitt A, Imarom S, Joachim A, Daugschies A. Interactive classification of porcine Eimeria spp. by computer-assisted image analysis. Vet Parasitol. 1999; 86(2): 105-112.

37. Katz N, Chavez A, Pellegrino J. A simple device for quantitative stool thick-smear technique in schistosomiasis mansoni. Rev Inst Med Trop São Paulo. 1972; 14(6): 397-400.

38. Dąbrowska J, Zdybel J, Karamon J, Kochanowski M, Stojecki K, Cencek T, Kłapeć T. Assessment of the nematode eggs (genera: Ascaris, Toxocara, Trichuris) in sewage sludge with the use of LIVE/DEAD Bacterial Viability Kit. Ann Agr Env Med. 2014; 21(1): 35-41.

39. Karkashan A, Khallaf B, Morris J, Thurbon N, Rouch D, Smith SR, Deighton M. Comparison of methodologies for enumerating and detecting the viability of Ascaris eggs in sewage sludge by standard incubation-microscopy, the BacLight Live/Dead viability assay and other vital dyes. Water Res. 2015; 68: 533-544.

40. Dendukuri N, Joseph L. Bayesian approaches to modeling the conditional dependence between multiple diagnostic tests. Biometrics. 2001; 57(1): 158-167.

41. Nikolay B, Brooker SJ, Pullan RL. Sensitivity of diagnostic tests for human soil-transmitted helminth infections: a meta-analysis in the absence of a true gold standard. Int J Parasitol. 2014; 44(11): 765-774.

42. Kochanowski M, Karamon J, Dąbrowska J, Cencek T. Experimental estimation of the efficacy of the FLOTAC basic technique. J Parasitol. 2014; 100(5): 633-639.

43. Karamon J, Sroka J, Cencek T. Limit of detection of sedimentation and counting technique (SCT) for Echinococcus multilocularis diagnosis, estimated under experimental conditions. Exp Parasitol. 2010; 124(2): 244-246. 